\title{
Valor predictivo de los factores de riesgo propuestos para el diagnóstico de enfermería: «Riesgo de caídas» en la población geriátrica institucionalizada
}

\author{
R. López-López a L. Lorenzo-López a , C.M. García-Martínez ${ }^{a}$, A. Maseda a , J.M. \\ Cancela-Carral $^{\mathrm{b}}$, J.C. Millán-Calenti ${ }^{\mathrm{a}}$ \\ ${ }^{a}$ Universidade da Coruña, Grupo de Investigación en Gerontología, Instituto de Investigación Biomédica de \\ A Coruña (INIBIC), Complexo Hospitalario Universitario de A Coruña (CHUAC), SERGAS, A Coruña, \\ España \\ ${ }^{b}$ Universidade de Vigo, Grupo de Investigación HealthyFit (HI22), Departamento de Didácticas Especiales, \\ Área de Educación Física y Deportiva, Vigo, Pontevedra, España
}

Objetivos: A fin de etiquetar el diagnóstico enfermero «Riesgo de caídas», la taxonomía NANDA-International (NANDA-I) propone categorizar los factores de riesgo en: adulto, ambiente, estado cognitivo, fisiológicos y de causa medicamentosa. Los objetivos de este trabajo han sido describir la prevalencia de caídas en personas mayores institucionalizadas, así como evaluar la frecuencia de aparición y el nivel de precisión diagnóstica de los factores de riesgo del mencionado diagnóstico.

Métodos: Se realizó un estudio observacional para evaluar la presencia o ausencia de 29 indicadores clínicos (factores de riesgo) del diagnóstico enfermero «Riesgo de caídas», de acuerdo a los criterios validados por la clasificación NANDA-I, en una muestra de 145 personas de $\geq 65$ años $(85,1 \pm 7,9)$ institucionalizadas en un complejo gerontológico. Para el cálculo de la precisión estadística de los factores de riesgo se realizaron medidas de análisis de test diagnóstico: sensibilidad, especificidad, valor predictivo positivo/negativo, eficiencia, odds ratio (OR) y curva ROC.

Resultados: La prevalencia de caídas fue del 37,2\%. El factor de riesgo más prevalente fue el de consumo de fármacos $(95,3 \%)$; no obstante, el uso de sujeciones y el déficit propioceptivo fueron los únicos factores de riesgo significativos. El área bajo la curva ROC para el uso de sujeciones fue del 0,63 , mientras que para el déficit propioceptivo fue del 0,58 , con una sensibilidad del 44 y del $19 \%$, una especificidad del 82 y del $97 \%$ y un OR de 6,25 y de 31,55 , respectivamente. El resto de indicadores analizados no resultaron específicos ni predictivos del diagnóstico enfermero «Riesgo de caídas».

Conclusiones: Los datos obtenidos sugieren que el uso de sujeciones y el déficit propioceptivo son buenos indicadores clínicos del riesgo de caídas en personas mayores institucionalizadas. Dado que los diagnósticos enfermeros orientan la planificación de intervenciones preventivas y la elaboración de los planes de cuidados, profundizar en el conocimiento de los factores de riesgo en las instituciones disminuirá la casuística de caídas, así como su morbimortalidad y coste sociosanitario asociado.

Este trabajo ha sido financiado por la Xunta de Galicia (ED431C2017/49 y Red FrailNet IN607C 2016/08). 